\title{
Robust 3D Segmentation of Anatomical Structures with Level Sets
}

\author{
C. Baillard and C. Barillot \\ IRISA-INRIA/CNRS, Campus de Beaulieu, \\ 35042 Rennes cedex, France \\ \{Caroline.Baillard,Christian.Barillot\}@irisa.fr
}

\begin{abstract}
This paper is concerned with the use of the level set formalism to segment anatomical structures in 3D medical images (ultrasound or magnetic resonance images). A closed 3D surface propagates towards the desired boundaries through the iterative evolution of a 4D implicit function. The major contribution of this work is the design of a robust evolution model based on adaptive parameters depending on the data. First the iteration step and the external propagation force, both usually constant, are automatically computed at each iteration. Additionally, region-based information rather than the gradient is used, via an estimation of intensity probability density functions over the image. As a result, the method can be applied to various kinds of data. Quantitative and qualitative results on brain MR images and 3D echographies of carotid arteries are discussed.
\end{abstract}

\section{Introduction}

The 3D segmentation of anatomical structures is crucial for many medical applications, both for visualization and clinical diagnosis purposes. Due to the huge amount of data and the complexity of anatomical structures, manual segmentation is extremely tedious and often inconsistent. Automatic segmentation methods are required to fully exploit $3 \mathrm{D}$ data. It is a very challenging task because they can usually not rely on image information only. Anatomical tissues are generally not homogeneous and their boundaries are not clearly defined in the images. It is therefore often necessary to involve prior knowledge about the shape or the radiometric behaviour of the structure of interest.

Deformable models define a powerful tool to accurately recover a structure using very few assumptions about its shape [15]. Such a model iteratively evolves towards the desired location according to a global energy minimization process. The functional energy is based on external forces derived from the data and internal forces related to the geometry of the contour. The limitations of this approach are well-known: the contour must be initialized close to the desired boundaries, and it can not cope with significant protusions nor topological changes. In the last few years, segmentation methods based on level sets have become very popular because they overcome classical limits of deformable models $[11,9,3]$. The evolving surface can change topology and cope with complex geometry, and the result is less dependent on initialization than with any other iterative method. This kind of approach has already been applied within a wide range of applications in computer vision. However, parametrization is still a limitation for practical use. Several evolution models have been proposed, but most of them include many 
parameters to be tuned: iteration step, weighting parameters, constant propagation term, etc. The tuning of these parameters determines the success of the method.

This paper describes a robust evolution model which enables a volume to be segmented with almost no parameter setting. It involves adaptive parameters depending on the data, and it relies on region-based information rather than gradient, via an estimation of intensity probability density functions over the image. The versatility of the segmentation is demonstrated on both brain structures in MR images and carotid arteries in 3D echography. Our strategy is presented in section 2. The two main stages of the method - intensity distribution analysis and surface evolution - are described in sections 3 and 4. Experimental results are presented and discussed in section 5.

\section{Segmentation Strategy Based on Level Sets}

Within the level set formulation [11], the evolving surface $S(t)$ is processed as a propagating front embedded as the zero level of a 4D scalar function $\Psi(\mathbf{x}, t)$. This hypersurface is usually defined as the signed distance from $\mathrm{x}$ to the front $S$ (negative inside the object). The evolution rule for $\Psi$ is:

$$
\frac{\partial \Psi}{\partial t}+F|\nabla \Psi|=0,
$$

where $F$ is a scalar velocity function depending on the local geometric properties of the front (local curvature) and external parameters related to the input data (image gradient for instance). The hypersurface $\Psi$ deforms iteratively according to $F$, and the position of the 3D front $S(t)$ is deduced from $\Psi$ at each iteration step using the relation $\Psi(\mathbf{x}(t), t)=0$. Practically, the hypersurface $\Psi^{n+1}$ at step $n+1$ is computed from $\Psi^{n}$ at step $n$ using the relation:

$$
\Psi^{n+1}(\mathbf{x})=\Psi^{n}(\mathbf{x})-\Delta t . F\left|\nabla \Psi^{n}(\mathbf{x})\right|, \quad \forall \mathbf{x} \in \mathbb{R}^{3}
$$

The design of the velocity function $F$ plays the major role in the evolution process. Several formulations have been proposed $[6,3,13,18]$. We have chosen the original formulation given by Malladi et.al. [9] for its simplicity:

$$
F=h_{I}(\nu-\rho \kappa)
$$

The term $\nu$ represents an external propagation force which makes the surface contract or expand. The parameter $\kappa$ represents the local curvature of the front and acts as a regularization term. The weighting $\rho$ expresses the importance given to regularization. Finally, the term $h_{I}$ is the data consistency term: it depends on the intensity I of the input data, and acts as a stopping factor at the location of the desired boundaries.

In the following, our definition of the parameters introduced in equations (2) and (3) is presented. They are defined with respect to the intensity probability density functions (PDFs) estimated inside and outside the structure. These PDFs will be respectively denoted $p_{i}(I)$ and $p_{e}(I)$, and the prior probability for a voxel to be inside the structure will be denoted $\alpha_{i}$. Our general strategy is illustrated in figure 1. The PDFs are first estimated by using both the initial segmentation and given statistical distribution models. This allows the parameters of the velocity function to be computed for the evolution of the hypersurface. The two stages are further described in the next two sections. 


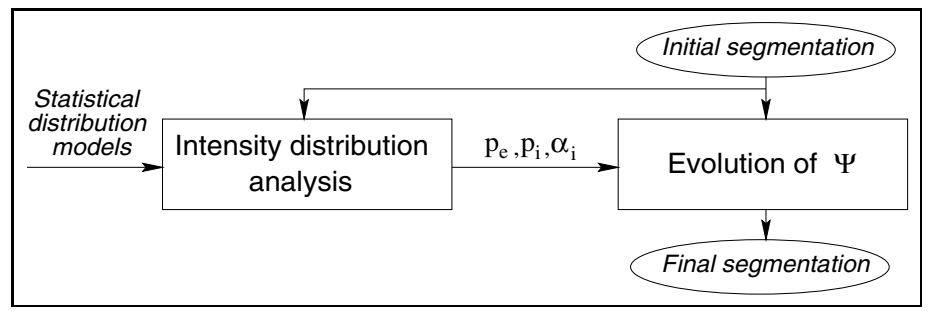

Fig.1. Strategy for 3D segmentation. The velocity function controlling the surface evolution is computed according to the estimated intensity density functions inside and outside the structure.

\section{Estimation of Intensity Probability Density Functions}

We assume that the intensity distribution of the input image can be written as a generalized mixture of $n$ kernels. Furthermore, these kernels do not necessarily have the same form. In this work, we have considered two kinds of laws: the Gaussian distribution and the shifted Rayleigh distribution.

Each component $k$ is associated to a class labelled by $\lambda_{k}$ and described by the variable $\Phi_{k}$, which characterizes the kernel distribution and the prior probability $P\left(\lambda_{k}\right)=$ $\pi_{k}$ of a voxel belonging to the class $\lambda_{k}$. The distribution mixture is thus described by the variable $\Phi=\left\{\Phi_{k}, 1 \leq k \leq n\right\}$. We begin by estimating $\Phi$ using the Stochastic Expectation-Maximisation (SEM) algorithm, which is a stochastic version of the EM algorithm [5]. Whilst the SEM algorithm is less sensitive to initialization than the EM algorithm, it is not guaranteed to find a local maximum of the likelihood surface, and the initialization of $\Phi_{k}$ is still important. For this initialization, we have distinguished two cases according to the value of $n$.

If $n=2$, the distribution mixture is bimodal, i.e., the object to be segmented and its background can each be approximated by a monomodal distribution. In this case, the surface initializing the segmentation process is used to produce two independent histograms from the input image: one for the initial segmentation and one for its background. The variables $\Phi_{0}$ and $\Phi_{1}$ characterizing the bimodal mixture are independently estimated over these complete data, providing two coarse estimates $\hat{\Phi}_{0}$ and $\hat{\Phi}_{1}$. This implicitely assumes that the intensity distribution inside and outside the initial segmentation are somehow representative of the distributions to be estimated. The bimodal approach is depicted in figure 2. Figure 3 shows an example of PDFs estimated with this method for the image shown in figure 6 . The bimodal model is not perfect, but it is good enough to separate the two main classes of the image.

If the distribution is not bimodal (case of brain MR images, see figure 5 for instance), the local maxima of the histogram are used as an initial guess for the center of the kernels, and the prior probabilities $\pi_{k}$ are initialized equal to each other. This initial guess of $\Phi$ is then processed by the SEM algorithm, providing an estimation of $\pi_{k}$ and $P\left(I \mid \lambda_{k}\right)$ for each class $\lambda_{k}$. Among these $n$ classes, only one or two characterize the structure to be segmented; the set of these classes will be noted $\Lambda_{i}$, and the complementary set of the classes exterior to the object will be noted $\Lambda_{e}$. The classes defining $\Lambda_{i}$ can be given as input parameters of the algorithm (number of classes and 


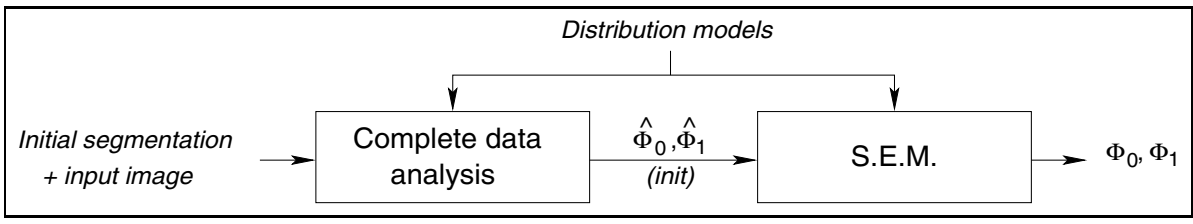

Fig.2. Estimation of the mixture model parameters when the distribution is bimodal.

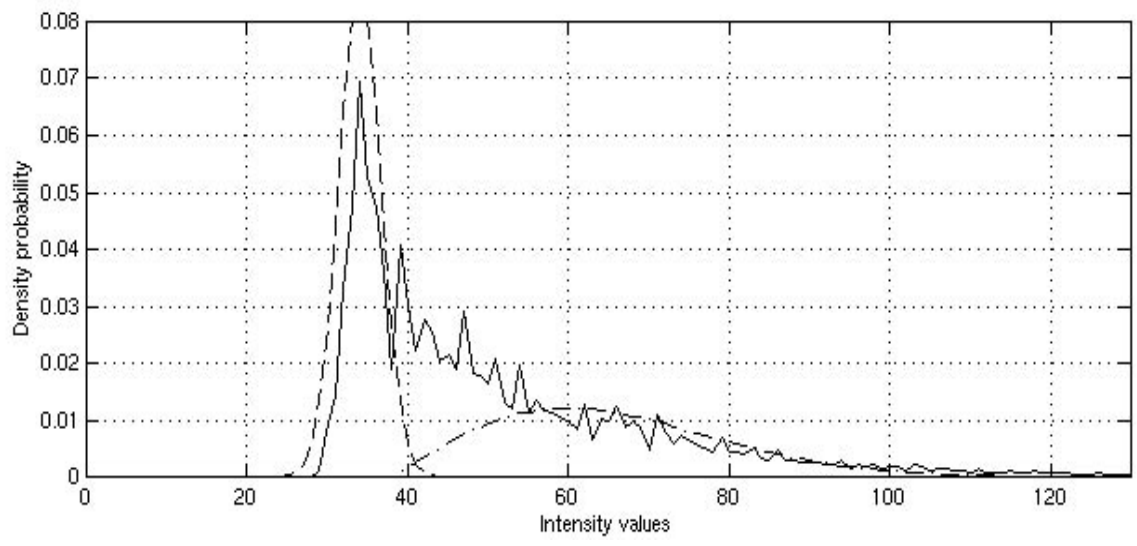

Fig.3. Estimation of the intensity mixture model parameters on the ultrasound image of figure 6 (bimodal case). The solid line represents the normalized histogram of the input image. The dash line is the estimated PDF inside the carotid arterie and the dashdot line is the estimated PDF outside it.

approximative mean value). Alternatively they can be automatically determined from the initialization of the surface (required for the segmentation process): only the classes highly represented inside the initial surface are said to belong to $\Lambda_{i}$. Once the sets $\Lambda_{i}$ and $\Lambda_{e}$ have been defined, the estimated intensity distributions inside and outside the object can be defined as:

$$
\left\{\begin{array}{l}
p_{i}(I)=\sum_{k / \lambda_{k} \in \Lambda_{i}} \pi_{k} P\left(I \mid \lambda_{k}\right) \\
p_{e}(I)=\sum_{k / \lambda_{k} \in \Lambda_{e}} \pi_{k} P\left(I \mid \lambda_{k}\right),
\end{array}\right.
$$

and the prior probability for a voxel to be inside the structure:

$$
\alpha_{i}=\sum_{k / \lambda_{k} \in \Lambda_{i}} \pi_{k}
$$

An example of estimated multimodal distribution mixture is shown in figure 4.

The SEM algorithm does not guarantee the optimal solution, but in practice any initial partition roughly representative of the inner and the outer distribution leads to a correct solution. In particular, the initial surface does not need to be close to the real boundaries. 


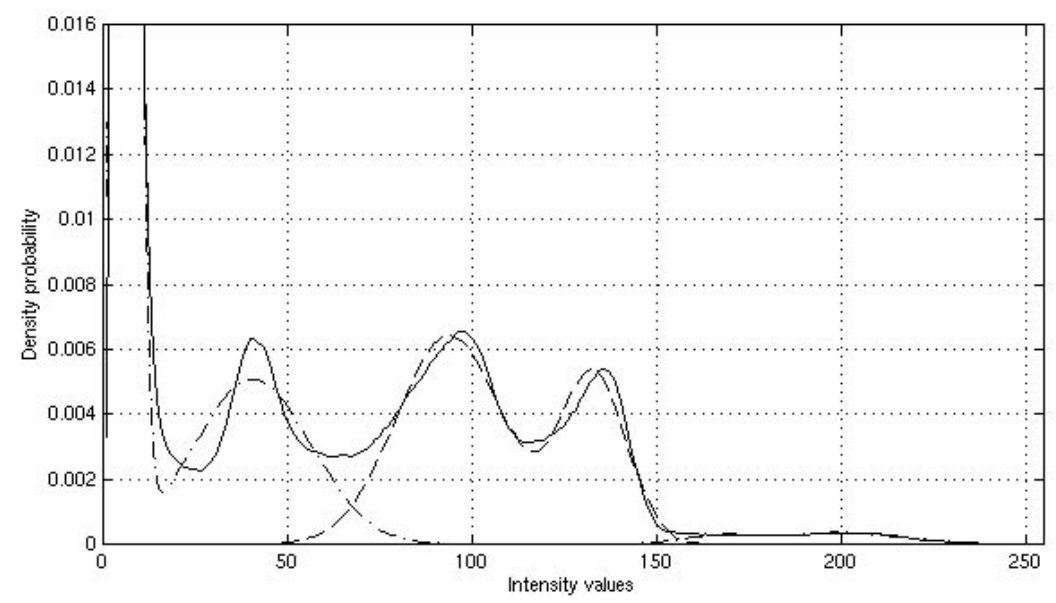

Fig.4. Estimation of the intensity mixture model parameters on brain MRI. The solid line represents the normalized histogram of the input image. The dash line is the estimated PDF inside the brain and the dashdot line is the estimated PDF outside the brain.

\section{Evolution Model}

\subsection{External Propagation Term $\nu$}

In equation (3), the sign of $\nu$ determines the direction of the external propagation force. Several approaches to 3D segmentation using this evolution model have imposed a oneway propagation force $\nu$, which either contracts or expands the whole surface all along the process $[8,18]$. However, when the initial position of the surface can be predicted (by tracking or by registration with an atlas for instance), the predicted and real positions usually overlap. It is therefore necessary to let the surface evolve in both directions. Some propagation models have been designed in order to solve this problem in $2 \mathrm{D}$, by involving a local analysis of intensity $[12,1,17]$.

The problem can be expressed as the classification of each point of the current interface $S(t)$. If a point belongs to the object then the surface should locally extend; if it does not, the surface should contract. We perform this classification by maximizing the a posteriori segmentation probability $p(\lambda \mid I)$, where $\lambda$ denotes the appartenance class of the considered point. According to Bayes rule, the maximization of the posterior distribution $p(\lambda \mid I)$ is equivalent to the maximization of $p(\lambda) p(I \mid \lambda)$, where $p(\lambda)$ is the prior of the class $\lambda$ and $p(I \mid \lambda)$ is the likelihood of intensity. The propagation term $\nu$ has then been defined as:

$$
\nu=\operatorname{Sign}\left\{\alpha_{i} p_{i}(I)-\left(1-\alpha_{i}\right) p_{e}(I)\right\}
$$

The propagation term $\nu$ is therefore positive if $p\left(\lambda_{i} \mid I\right)>p\left(\lambda_{e} \mid I\right)$. The point is more likely to be inside the object than outside, and the surface extends. Note, we always have $|\nu|=1$. Experiments with a continous-varying function have not shown any improvement. Besides, this simple definition needs no tuning. 


\subsection{Curvature Term $\rho \kappa$}

The regularization parameter $\kappa$ at a point $\mathbf{x}$ is the curvature of the interface, computed at the closest point to $\mathrm{x}$. This curvature is computed using the partial derivatives of $\Psi$.

The respective roles of the propagation and the regularization terms are entirely determined by the weight $\rho$, which makes the process very easy to tune. The weighting parameter $\rho$ can be interpreted as the particular curvature radius leading to a stable position $(F=0)$.

\subsection{Stopping Factor $h_{I}$}

The stopping factor is a data consistency term traditionally related to the intensity gradient $\nabla I$ of the input image [9]. Since this gradient is only defined for points belonging to the interface (zero level set of the hypersurface), an extended gradient function needs to be defined over $\mathbb{R}^{3}$ by the intensity gradient of the closest neighbour on the interface. However, gradient information has no meaning for very noisy and/or low-contrasted images (like ultrasound data). Besides, high gradients do not necessarily indicates a relevant boundary between the structure to be segmented and its background.

Similarly to the approach described in [14] in the 2D case, we have related this term to the posterior probability of having a transition between the object and its background. Let $\mathbf{x}$ be a voxel of the current interface, and $\lambda$ the estimated class of $\mathbf{x}$; the parameter $\lambda$ is supposed to be known here because it is taken into account via the sign of $\nu$. The posterior probability of $\mathbf{x}$ being a transition, given $I$ and $\lambda$, is given by:

$$
p_{T}(\mathbf{x} \mid I, \lambda)= \begin{cases}p(\mathbf{x}-\mathbf{n} \in \overline{\mathcal{O}} \mid I) & \text { if } \lambda \in \Lambda_{i} \\ p(\mathbf{x}-\mathbf{n} \in \mathcal{O} \mid I) & \text { if } \lambda \in \Lambda_{e},\end{cases}
$$

where $\mathbf{n}=\frac{\Delta \Psi}{\Delta \Psi}$ is the current normal vector of the hypersurface, $\mathcal{O}$ is the object to be segmented and $\overline{\mathcal{O}}$ its complementary. If $\nu>0$, the posterior transition probability of $\mathbf{x}$ is the probability of the neighbouring voxel $\mathbf{x}^{\prime}=\mathbf{x}-\mathbf{n}$ (located outside the current volume) to be located outside the object to be segmented. Using Bayes rule, this transition probability can be expressed as :

$$
p_{T}(\mathbf{x} \mid I, \lambda)= \begin{cases}\frac{\left(1-\alpha_{i}\right) p_{e}\left(I\left(\mathbf{x}^{\prime}\right)\right)}{\alpha_{i} p_{i}\left(I\left(\mathbf{x}^{\prime}\right)\right)+\left(1-\alpha_{i}\right) p_{e}\left(I\left(\mathbf{x}^{\prime}\right)\right)} & \text { if } \lambda \in \Lambda_{i} \\ \frac{\alpha_{i} p_{i}\left(I\left(\mathbf{x}^{\prime}\right)\right)}{\alpha_{i} p_{i}\left(I\left(\mathbf{x}^{\prime}\right)\right)+\left(1-\alpha_{i}\right) p_{e}\left(I\left(\mathbf{x}^{\prime}\right)\right)} & \text { if } \lambda \in \Lambda_{e}\end{cases}
$$

The stopping factor $h_{I}$ at a point $\mathrm{x}$ belonging to the interface is finally defined as a decreasing function of $p_{T}(\mathbf{x} \mid I, \lambda)$. Since this probability is only defined on the interface, it is extended to $\mathbb{R}^{3}$ via the closest point on the current interface:

$$
h_{I}(\mathbf{x})=g\left(p_{T}(\tilde{\mathbf{x}} \mid I, \lambda)\right) \quad \forall \mathbf{x} \in \mathbb{R}^{3},
$$

where $g$ is decreasing from $[0 ; 1]$ to $[0 ; 1]$, and $\tilde{\mathbf{x}}$ is the closest point to $\mathbf{x}$ on the interface.

\subsection{Iteration Step $\Delta t$}

The iteration step $\Delta t$ of equation (2) is usually constant and manually tuned. We propose to compute it automatically at each iteration in order to improve robustness. 
The stability of the process requires a numerical scheme for the computation of $\Delta \Psi$, called upwind sheme. This scheme induces a limit on the iteration step $\Delta t$, called the CFL restriction (Courant-Friedrichs-Levy). More precisely, writing equation (1) as $\Psi_{t}+H\left(\Psi_{x}, \Psi_{y}, \Psi_{z}\right)=0$, where $H$ is the Hamiltonian defined by:

$$
H(u, v, w)=\sqrt{u^{2}+v^{2}+w^{2}} \cdot F,
$$

the CFL restriction can be expressed in 3D [7] as:

$$
1 \geq \Delta t .\left(\frac{\left|H_{u}\right|}{\Delta x}+\frac{\left|H_{v}\right|}{\Delta y}+\frac{\left|H_{w}\right|}{\Delta z}\right),
$$

where $H_{u}, H_{v}, H_{w}$ denote the partial derivatives of $H$ with respect to $u, v, w$. Since we work with a regular sampling grid, we can assume $\Delta x=\Delta y=\Delta z=1$. According to equation (3) and the definition of the parameters involved, the velocity function $F$ is independent from $u, v, w$. The terms $h_{I}$ and $\nu$ only depends on the image data. The curvature term $\kappa$ is the curvature of the interface at the closest point, and it does not depend on the local characteristics of $\Psi$. Therefore the partial derivatives of $H$ can be directly computed from (10) at each iteration of the process, and the best value for $\Delta t$ which guarantees stability is given by:

$$
\tilde{\Delta} t=\min \left\{\frac{1}{F} \cdot \frac{\sqrt{u^{2}+v^{2}+w^{2}}}{|u|+|v|+|w|}\right\}
$$

\section{Experimental Results}

\subsection{Segmentation of Brain MR Images}

The segmentation algorithm was run on simulated data provided by the MNI [4]. They consist of a phantom collected with 3 levels of noise and inhomogeneity, associated to a reference classification. The method was applied to segment the whole brain (grey matter + white matter) and numerically assessed with overlapping measures: sensitivity, specificity, and total performance [16]. The segmentation was initialized with a cube $100 \times 70 \times 70$, and the classes of interest (grey matter + white matter) automatically determined as described in section 3. It was compared with a segmentation method based on morphological operators and tuned with a "best practice" parameter set. The numerical results of both methods are summarized in table 1. The total performance achieved with our algorithm is stable around $98.3 \%$, even in critical conditions $(9 \%$ noise and $40 \%$ inhomogeneity). This is far better than the other method which is very dependent on noise. The improvement mainly concerns the sensitivity of the detection.

Experiments have also been run on a database of $18 \mathrm{MR}$ images (volume size $256 \times 256 \times 176$ ). All the subjects have been processed in the same way, and using exactly the same parameter set. The iteration step is automatically computed, as well as the threshold on intensity values. The weighting parameter on regularization has been set to $\rho=3$ (see eq. 3), which has appeared to be a good compromise to avoid propagation out of the brain (possible when the cerebro-spinal fluid area is thiner than the image resolution), whilst preserving the highly convoluted characteristics of the cortical surface. Out of the 18 subjects, only 1 segmentation failed, and the failure could be detected because the propagation speed started to increase (it should keep decreasing). 


\begin{tabular}{|l|cccc|ccc|}
\cline { 2 - 7 } \multicolumn{1}{c|}{} & \multicolumn{4}{|c|}{ Levels sets } & \multicolumn{3}{|c|}{ Morphological filtering } \\
\cline { 2 - 7 } \multicolumn{1}{c|}{} & Nb iter & Sens. & Spec. & Total & Sens. & Spec. & Total \\
\hline $3 \%$ noise, 0\% inhom. & 710 & $96.2 \%$ & $99.0 \%$ & $98.3 \%$ & $86.7 \%$ & $98.7 \%$ & $95.7 \%$ \\
3\% noise, 20\% inhom. & 680 & $96.3 \%$ & $98.9 \%$ & $98.2 \%$ & $83.5 \%$ & $99 \%$ & $95.2 \%$ \\
$9 \%$ noise, 40\% inhom. & 800 & $95.95 \%$ & $98.9 \%$ & $98.2 \%$ & $69.8 \%$ & $99.5 \%$ & $92.3 \%$ \\
\hline
\end{tabular}

Table1. Quantitative assessment on the phantom. The number of iterations, the sensitivity, specificity, and total performance measures are given for three levels of noise and two segmentation methods: the method based on level sets (initialization with a cube $100 \times 70 \times 70$ ) and a segmentation method based on morphological operators.

All the other 17 subjects were correctly segmented. Figure 5 shows the segmentation results on two different subjects. Protusions of brain and ventricles are properly recovered, despite of the surface being initialized far away from it. The method can naturally cope with change of topology: the ventricles inside the brain have also been recovered. On average, 1000 iterations are necessary to segment the whole brain on real data starting from a $100 \times 70 \times 70$ cube. The number of iterations can be reduced to 300 if the surface is initialized close to the real boundaries by a registration technique (see [2] for details).

\subsection{Segmentation of 3D Ultrasound Images}

The method has also been applied to 3D ultrasound images of carotid artery. This kind of images is difficult to segment automatically due to the speckle noise. The intensity distribution inside the carotid has been modelized by a Gaussian (shadow area), whereas the exterior is modelized by a Rayleigh distribution (reverberation area) [10]. We have used a weighting value of $\rho=10$ for regularization. All the other parameters are the same as for experiments on brain MRI. Figures 6 and 7 show the results of segmentation on two different images. They allow us to be very optimistic about the use of this statistical variational approach to segment 3D ultrasound images.

\section{Conclusion and Further Work}

This paper has presented a robust evolution model for segmenting structures in 3D images using the level set formalism. The level set approach is very appropriate to segment anatomical structures without strong prior information. The statistical analysis of intensity distributions provides relevant information about input data. The design of an adaptive evolution force and an adaptive iteration step provides a good trade-off between convergence speed and stability. Almost no parameter needs to be tuned, only the regularization weight $\rho$ has to be set depending on the data shape and noise. Good quality results have been produced on brain MRI and 3D echographies of carotid. The results demonstrate that a variational approach mixed with region-based analysis (statistical models) significantly improves the sensitivity and the robustness of the segmentation.

Thanks to the genericity of the method, the segmentation can be achieved for various image modalities and various kinds of anatomical structure. Our goal is now to focus on 3D ultrasound. Due to speckle noise, a method mixing variational and statistical models should be particularly appropriate to achieve good automatic segmentation. For 

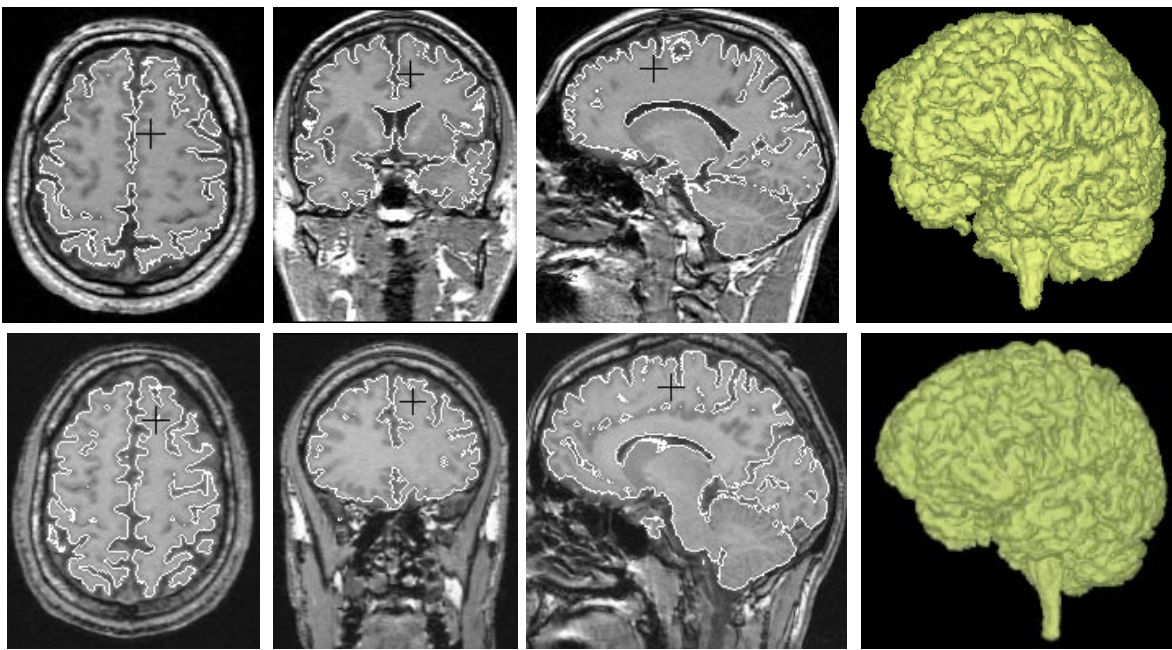

Fig.5. Brain segmentation for two different subjects (initialization with a cube of size $70 \times 100 \times 100$ located inside the brain). The first three columns respectively show axial, coronal and saggital planes, the last column shows 3-D views of the segmented brains.

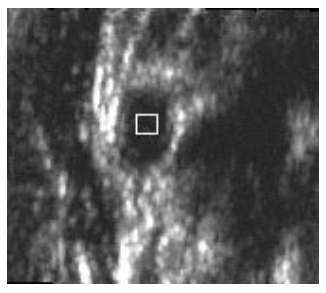

(a)

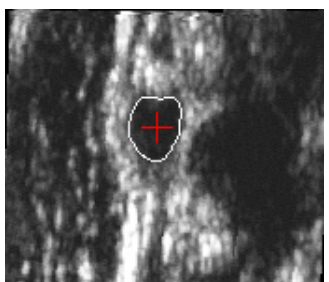

(b)

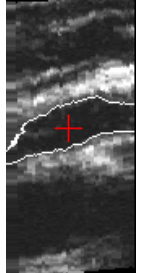

(c)

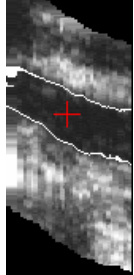

(d)

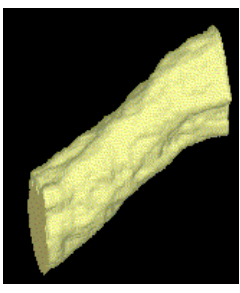

(e)

Fig.6. Segmentation result on a $256 \times 256 \times 80$ ultrasound image of carotid (isotropic voxels). (a): initialization of the segmentation with a $10 \times 10 \times 25$ cube. (b,c,d): final segmentation (three visualization planes). (e) 3D view of the segmented carotid.
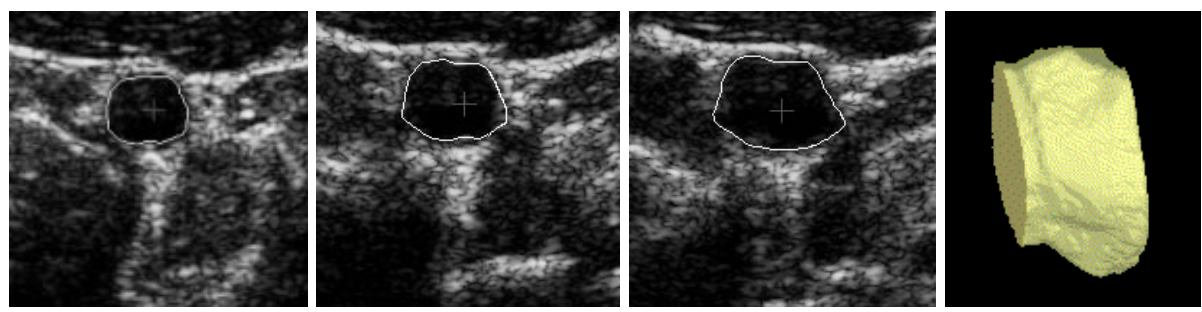

Fig.7. Three transaxial slices and a $3 \mathrm{D}$ view of a segmented subpart of carotid (non isotropic voxels). The $256 \times 256 \times 36$ input image was initialized with a $50 \times 50 \times 25$ cube inside the carotid. 
this purpose, it will be necessary to involve more prior information about the shape of the anatomic structure.

Acknowledgements. The authors would like to thank Drs L. Pourcelot and F. Tranquart from Tours university hospital and Dr A. Fenster from RRI (London, Ontario) for providing the US data, and the GIS "Sciences de la cognition" for granting the MR acquisition project.

\section{References}

[1] O. Amadieu, E. Debreuve, M. Barlaud, and G. Aubert. Inward and outward curve evolution using level set method. In ICIP, Kobe, Japan, oct. 1999.

[2] C. Baillard, P. Hellier, and C. Barillot. Segmentation of 3D brain structures using level sets and dense registration. In IEEE Workshop on Mathematical Methods in Biomedical Image Analysis, jun. 2000.

[3] V. Caselles, R. Kimmel, and G. Sapiro. Geodesic active contours. IJCV, 22:61-79, 1997.

[4] D.L. Collins, A.P. Zijdenbos, V. Kollokian, J.G. Sled, N.J. Kabani, C.J. Holmes, and A.C. Evans. Design and construction of a realistic digital brain phantom. IEEE Transactions on Medical Imaging, 17(3):463-468, jun. 1998.

[5] A.P. Dempster, N.M. Laird, and D.B. Rubin. Maximum likelihood from incomplete data via the em algorithm. In Royal Statistical Society, pages 1-38, 1976.

[6] J. Gomes and O. Faugeras. Reconciling distance functions and Level-Sets. Technical Report 3666, Inria, apr. 1999.

[7] R. Kimmel, N. Kiryati, and A. Bruckstein. Analyzing and synthesizing images by evolving curves with the Osher-Sethian method. IJCV, 24(1):37-55, 1997.

[8] L. M. Lorigo, O. Faugeras, W. E. L. Grimson, R. Keriven, and R. Kikinis. Segmentation of bone in clinical knee MRI using texture-based geodesic active contours. In A. Colchester and S. Delp, editors, MICCAI, number 1496 in LNSC, pages 1195-1204, Cambridge, MA, USA, oct. 1998. Springer.

[9] R. Malladi, J.A. Sethian, and B.C. Vemuri. Shape modeling with front propagation: A level set approach. IEEE Tr. on PAMI, 17(2):158-175, feb. 1995.

[10] M. Mignotte and J. Meunier. Deformable template and distribution mixture-based data modeling for the endocardial contour tracking in an echographic sequence. In $C V P R$, pages 225-230, jun. 1999.

[11] S. Osher and J.A. Sethian. Fronts propagating with curvature dependent speed: Algorithms based on Hamilton-Jacobi formulation. J. of Computational Physics, 79:12-49, 1988.

[12] C. Papin, P. Bouthemy, E. Mémin, and G. Rochard. Tracking and characterization of convective clouds from satellite images. In EUMETSAT Meteo. Satellite Data Users Conf., Copenhague, sep. 1999.

[13] N. Paragios and R. Deriche. Unifying boundary and region-based information for geodesic active tracking. In CVPR, volume 2, pages 300-305, Fort Collins, Colorado, jun. 1999.

[14] N. Paragios and R. Deriche. Video and image sequence analysis - geodesic active contours and level sets for the detection and tracking of moving objects. IEEE Tr. on PAMI, 22(3):266-280, 2000.

[15] D. Terzopoulos. Regularization of inverse visual problems involving discontinuities. IEEE Tr. on PAMI, 8(2):413-424, 1986.

[16] JH. Van Bemmel and MA. Musen. Handbook of medical informatics. Springer, URL : http://www.mieur.nl/mihandbook, 1997.

[17] A. Yezzi, A. Tsai, and A. Willsky. Binary and ternary flows for image segmentation. In ICIP, Kobe, Japan, oct. 1999.

[18] X. Zeng, L.H. Staib, R.T. Schultz, H. Tagare, L. Win, and J.S. Duncan. A new approach to 3D sulcal ribbon finding from MR images. In C. Taylor and A. Colchester, editors, MICCAI, number 1679 in LNSC, pages 148-157. Springer, sep. 1999. 